\title{
SUBMILLIMETRE SPECTRAL INDICES OF RADIO-QUIET QUASARS
}

\author{
D. H. HUGHES \\ Astrophysics, Dept. of Physics., Oxford University, U.K. \\ J. S. DUNLOP \\ Dept. of Chemical \& Physical Sciences, Liverpool John Moores University, U.K. \\ E. I. ROBSON \\ Joint Astronomy Centre, Hilo, Hawaii, U.S.A. \\ and \\ W. K. GEAR \\ Royal Observatory, Edinburgh, U.K.
}

Hughes etal. (1993) have made submillimetre continuum observations of 10 IRAS selected radio-quiet quasars (RQQs). Three RQQs, I Zw 1, Mrk1014 and Mrk376, have been detected at 800 and $450 \mu m$ using the ${ }^{3} \mathrm{He}$ bolometer UKT14 on the 15-m James Clerk Maxwell telescope. These submillimetre data, together with existing $1.3 \mathrm{~mm}$ observations (Chini etal. 1989) demonstrate that the measured submillimetre spectral indices, $\left\langle\alpha_{s m}\right\rangle=3.8 \pm 0.5$, significantly exceed the critical theoretical limit of $\alpha_{s m}=2.5$ predicted for the self-absorption of synchrotron emission. This result is independent of any contributions to the $100 \mu \mathrm{m}$ IRAS fluxes from cirrus emission in the host galaxies, extended circumnucl ear starformation and FIR emission from companion or confusing sources. All current non-thermal models (de Kool \& Begelman 1989, Schlickeiser etal. 1991) are rejected in favour of the alternative explanation that the FIR luminosity is dominated by thermal emission from warm $(45-60 \mathrm{~K})$ dust grains. The submillimetre optical depth and source-size for the thermal emission cannot yet be constrained by these data and, as a result, no discrimination can be made between dust heated by an extended ( $>1 \mathrm{kpc}$ ) starburst region or a central compact luminosity source. However ground-based imaging observations at mid-IR wavelengths and FIR photometry $(60-200 \mu m)$ with the KAO are currently in progress specifically to address this problem. The high gas masses $\left(>10^{10} M_{\odot}\right)$ in RQQs inferred from the submillimetre continuum observations are in agreement with the $\mathrm{H}_{2}$ masses determined from $\mathrm{CO}$ measurements. Alternatively the results show that the $\mathrm{M}_{H_{2}} / \mathrm{M}_{\text {dust }}$ ratio measured in RQQs $(\sim 370 \pm 150)$ is consistent with that measured in spiral galaxies and ultra-luminous IRAS galaxies.

\section{References}

Chini,R., Kreysa,E. \& Biermann,P.L., 1989, A\&A, 219, 87

de Kool,M. \& Begelman,M.C. 1989. Nature, 338, 484

Hughes,D.H., Robson,E.I., J.S. Dunlop \& Gear,W.K., 1993. MNRAS, 263, 607

Schlickeiser,R., Biermann,P.L. \& Crusius-Wätzel,A., 1991. A\&A, 247, 283

324

T. J.-L. Courvoisier and A. Blecha: Multi-Wavelength Continuum Emission of AGN, 324.

(C) 1994 IAU. Printed in the Netherlands. 